\title{
Monetäre Indikatoren
}

Wie bereits in Kapitel 2 erwähnt, sind die FuE-Ausgaben bzw. FuE-Aufwendungen einer der beiden Kernindikatoren der offiziellen FuE-Erhebung. Monetäre Indikatoren haben auch bei der Innovationserhebung einen hohen Stellenwert. Als Vorteile monetärer Indikatoren wurden bereits genannt:

1. Anwendbarkeit in allen Sektoren

2. Vollständige und überschneidungsfreie Erfassung aller FuE-Aktivitäten einschließlich der erfolglosen Forschung

3. Aggregierbarkeit

4. Kompatibilität mit anderen Erhebungen

Allerdings wurde auch schon darauf hingewiesen, dass die der FuE-Erhebung zugrunde liegende Hypothese „hohe Aufwendungen bedeuten auch viel FuE“ durchaus anzuzweifeln ist. Zum einen nimmt sie keine Rücksicht auf mögliche Produktivitätsunterschiede zwischen den Wissenschaftlerinnen und Wissenschaftlern. Zum anderen wird nicht berücksichtigt, dass Forschung in verschiedenen Bereichen unterschiedlich kostenintensiv ist.

Darüber hinaus ist zu berücksichtigen, dass die FuE-Aufwendungen, wie sie in der FuEErhebung erfasst und schließlich kommuniziert werden, nominale Größen sind. Das heißt, sie umfassen sowohl eine Preis- als auch eine Mengenkomponente. Da das politische Ziel aber in der Regel darin besteht, mehr FuE und nicht etwa teurere FuE zu bekommen, müssten die FuE-Aufwendungen deflationiert werden. Ein entsprechend geeigneter FuE-Deflator existiert aber in der amtlichen Statistik nicht. Die bekannten Deflatoren aus der Preisstatistik werden den Ansprüchen für FuE nur teilweise gerecht. Eine teilweise Lösung dieses Problems wird dadurch angestrebt, dass die internen FuE-Aufwendungen eines Landes in Relation zum Bruttoinlandsprodukt gesetzt werden (BIP-Relation). Das ermöglicht nicht nur eine Vergleichbarkeit von Ländern verschiedener Größe bzw. Wirtschaftskraft, sondern verringert auch das Inflationsproblem. Allerdings sind die Preissteigerungen im Zähler (interne FuE-Aufwendungen) und im Nenner (nominales BIP) von unterschiedlicher Qualität, sodass das Deflationierungsproblem nur teilweise gelöst wird.

Neben der Veränderung des Preisniveaus (Inflation) stellt auch die unterschiedliche Höhe von Preisen und Löhnen in verschiedenen Ländern ein Problem dar. So weist ein Land mit hohen Lohn- und Lohnnebenkosten bei der gleichen Anzahl an FuE-Beschäftigten höhere Personalausgaben (und damit höhere Gesamtausgaben) aus. In der Logik der FuE- und der Innovationserhebung heißt das, dass in diesem Land dann auch „mehr“ FuE betrieben wird. Diese Schlussfolgerung wurde aber bereits in Kapitel 2 als diskussionswürdig bezeichnet. 


\section{Übersicht der Indikatoren}

\section{Indikatoren für alle Sektoren}

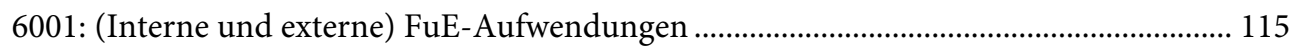

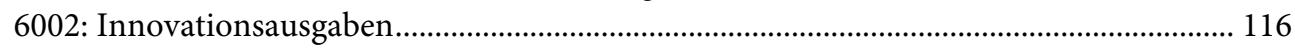

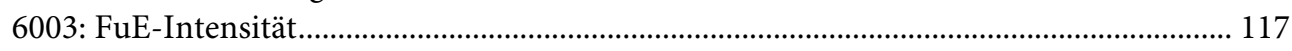

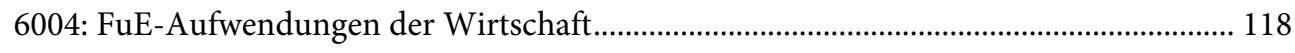

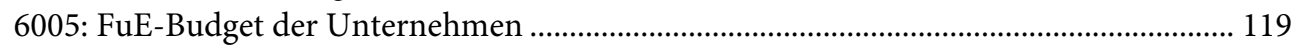

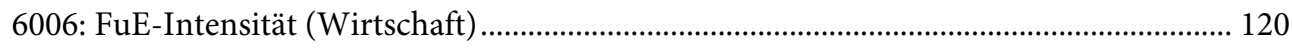

6007: Globale FuE-Aufwendungen multinationaler Unternehmen .................................... 121

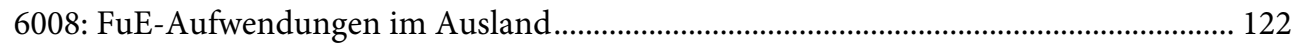

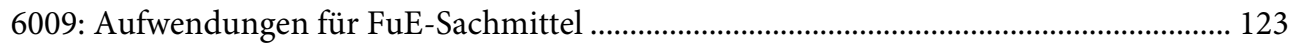

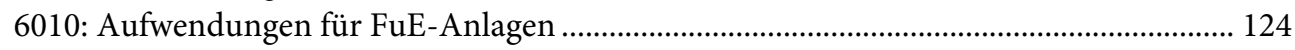

6011: Anteil der FuE-Aufwendungen an den Innovationsaufwendungen ........................... 125

6012: Staatsausgaben für Bildung, Forschung und Technologie ............................................ 126

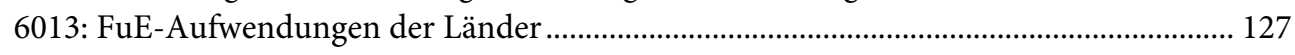

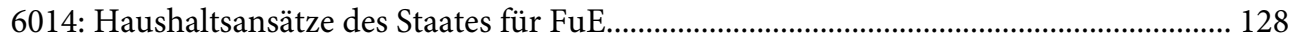

6015: FuE-Aufwendungen nach Art der Forschung .......................................................... 129

\section{Hochschulen}

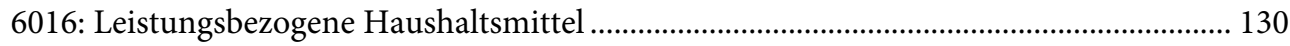

6017: Anteil der durch Unternehmen finanzierten FuE an Hochschulen ............................. 131

6018: Anteil der FuE-Ausgaben an den Gesamtausgaben der Hochschulen........................ 132

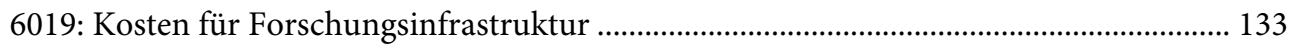

\section{FuE-/Innovationsförderung}

6020: B-Index für steuerfinanzierte FuE-Subventionen ...................................................... 134

6021: Staatlich geförderte FuE im Verhältnis zum nationalen oder regionalen BIP ........... 135

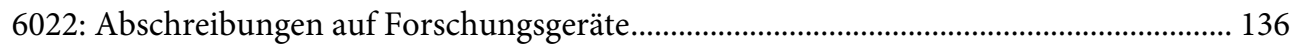

6023: FuE-Aufwendungen nach Herkunft der Mittel............................................................ 137

6024: Nutzung der staatlichen Innovationsförderung ............................................................. 138

6025: FuE-Aufwendungen pro Euro Steuergutschrift ............................................................ 139

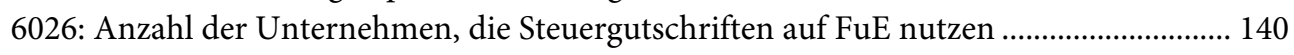

6027: Anzahl der Unternehmen, die FuE-Subventionen nutzen ....................................... 141

\section{Output-Indikatoren}

6028: Umsatzanteil durch neue und verbesserte Produkte ................................................. 142

\section{Negativindikatoren}

6029: Gründe, auf Innovationen zu verzichten. 


\section{Indikatoren für alle Sektoren}

ID: 6001

\section{Indikator:}

(Interne und externe) FuE-Aufwendungen

\section{Beschreibung:}

FuE-Aufwendungen setzen sich aus laufenden Aufwendungen für FuE und Bruttoanlageinvestitionen für in einem bestimmten Zeitraum durchgeführte FuE zusammen.

\section{Quelle (Beispiel):}

OECD (2015), Ziffer 4.10; Wissenschaftsstatistik (2019a), Abschnitt 3; Wissenschaftsstatistik (2019b); Destatis (2020)

\section{Anwendbar auf:}

Alle Sektoren

Eigenschaften des Indikators:

\begin{tabular}{|l|l|l|r|}
\hline Qualitativ & & $\mathrm{X}$ & Quantitativ \\
\hline Input & $\mathrm{X}$ & & Output \\
\hline Absolut & $\mathrm{X}$ & & Relativ \\
\hline
\end{tabular}

Sonstige Eigenschaften:

Aggregierbarkeit

$+$

Berücksichtigung erfolgloser Forschung

$+$

\section{Kommentare:}

Interne Forschung findet in der jeweiligen statistischen Einheit statt, externe Forschung sind Forschungsaufträge an andere Institutionen. Bei Unternehmen können das auch Unternehmen der eigenen Unternehmensgruppe sein.

Eine Summation von internen und externen FuE-Aufwendungen ist nur auf Mikrodatenebene möglich, weil es sonst zu Doppelzählungen kommt. 


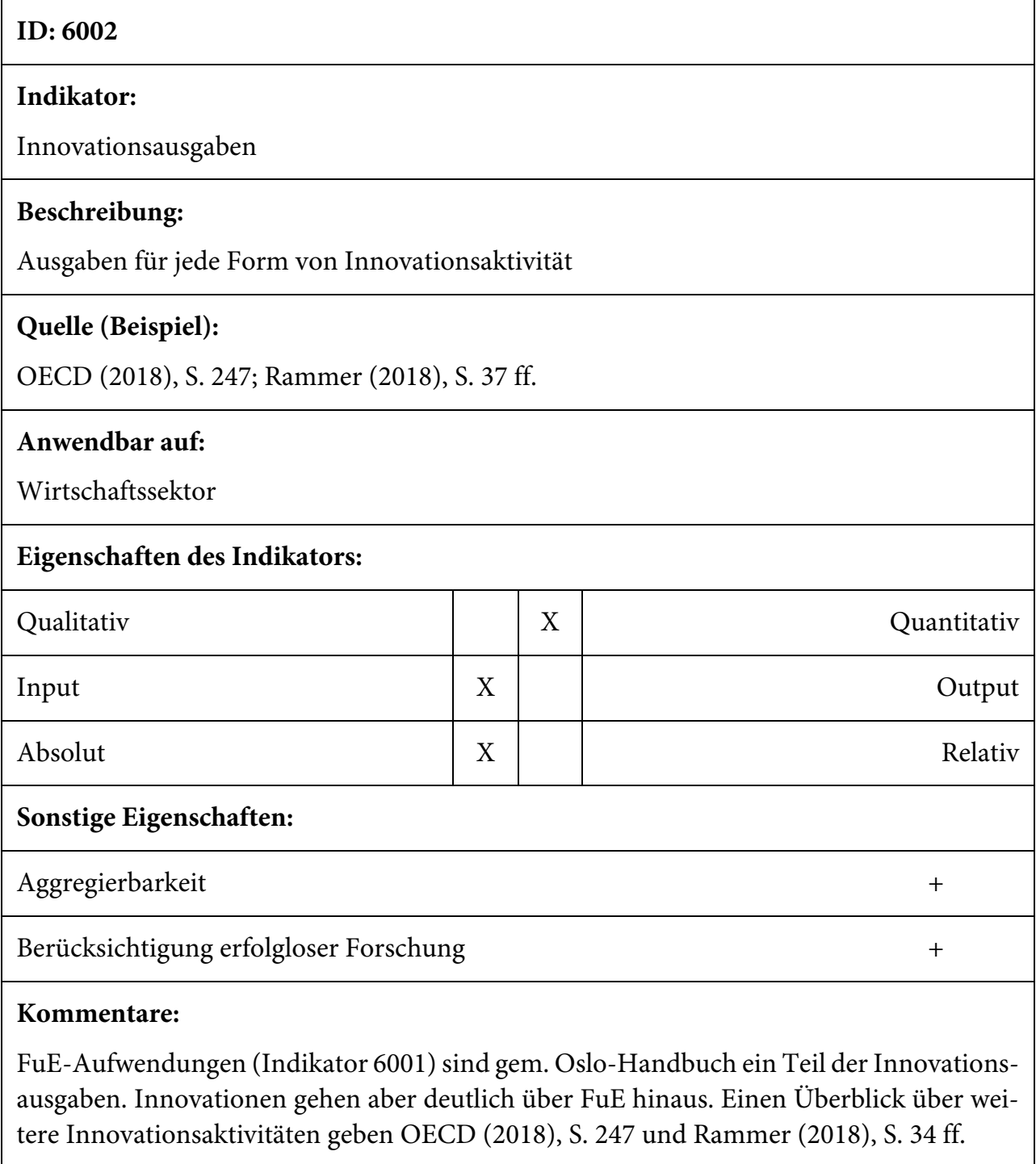


ID: 6003

Indikator:

FuE-Intensität

Beschreibung:

Interne FuE-Aufwendungen im Verhältnis zum (regionalen) Bruttoinlandsprodukt

Quelle (Beispiel):

Wissenschaftsstatistik (2019a, b); BMBF (2020a)

Anwendbar auf:

Alle Sektoren

Eigenschaften des Indikators:

\begin{tabular}{|l|l|l|r|}
\hline Qualitativ & & $\mathrm{X}$ & Quantitativ \\
\hline Input & $\mathrm{X}$ & & Output \\
\hline Absolut & & $\mathrm{X}$ & Relativ \\
\hline
\end{tabular}

Sonstige Eigenschaften:

Aggregierbarkeit $\quad 0$

Berücksichtigung erfolgloser Forschung +

\section{Kommentare:}

Der Indikator wird vor allem für die internationale und regionale Vergleichbarkeit der FuE-Aufwendungen verwendet. Ein ähnlicher, von Eurostat veröffentlichter Indikator ist das Verhältnis zwischen FuE-Aufwendungen und Bevölkerungszahl. Die Forschungsintensität ist z. B. Grundlage der Europa-2020-Strategie oder der High-Tech-Strategie. 


\section{ID: 6004}

\section{Indikator:}

FuE-Aufwendungen der Wirtschaft

\section{Beschreibung:}

FuE-Aufwendungen des Wirtschaftssektors gemäß der Abgrenzung des Frascati-Handbuchs

\section{Quelle (Beispiel):}

OECD (2015), Kap. 7; Wissenschaftsstatistik (2019a, b)

\section{Anwendbar auf:}

Wirtschaftssektor

Eigenschaften des Indikators:

\begin{tabular}{|l|l|l|r|}
\hline Qualitativ & & $\mathrm{X}$ & Quantitativ \\
\hline Input & $\mathrm{X}$ & & Output \\
\hline Absolut & $\mathrm{X}$ & & Relativ \\
\hline
\end{tabular}

Sonstige Eigenschaften:

Aggregierbarkeit

Berücksichtigung erfolgloser Forschung

$+$

\section{Kommentare:}

Vgl. Anmerkungen in Kapitel 2 


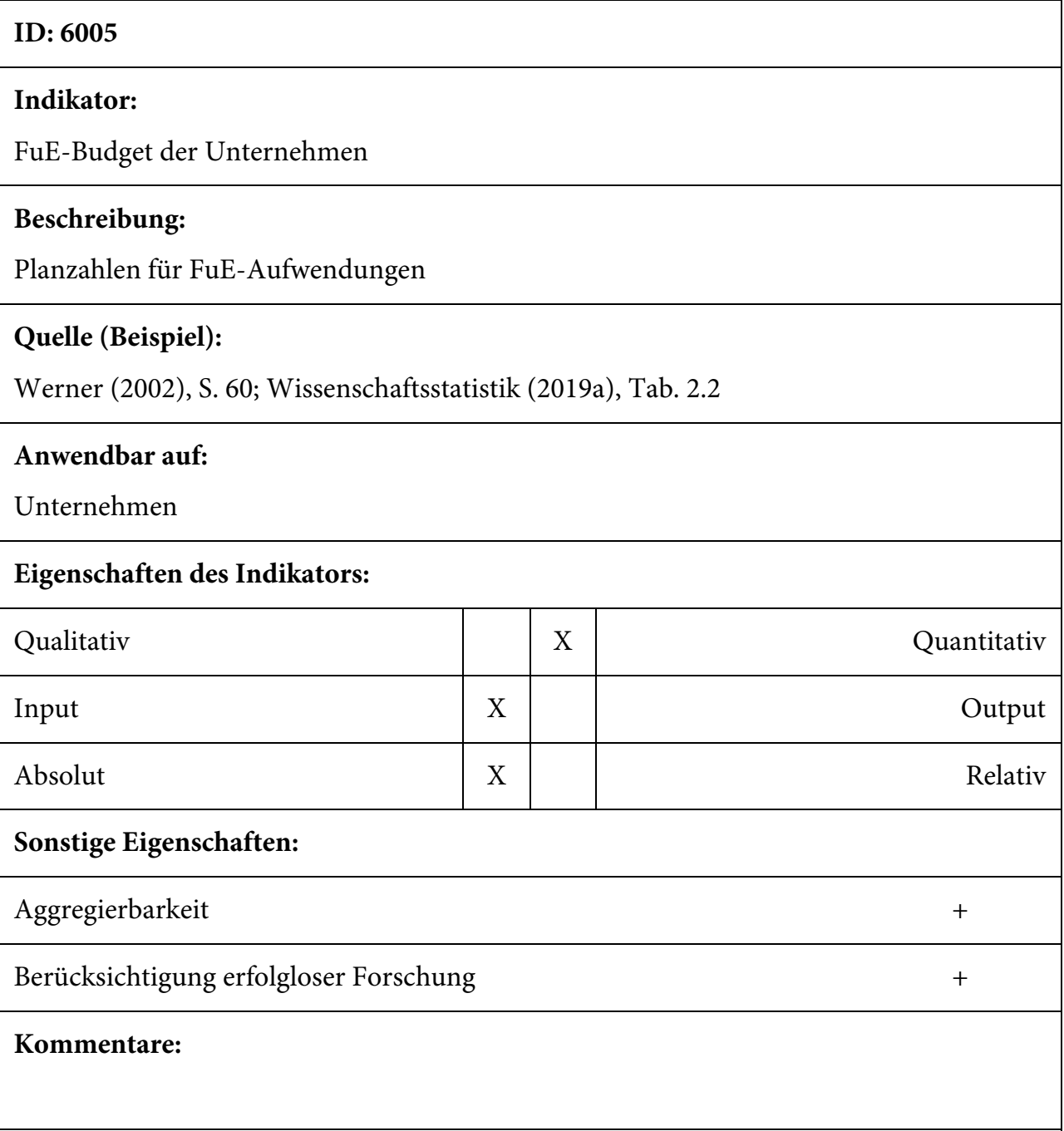




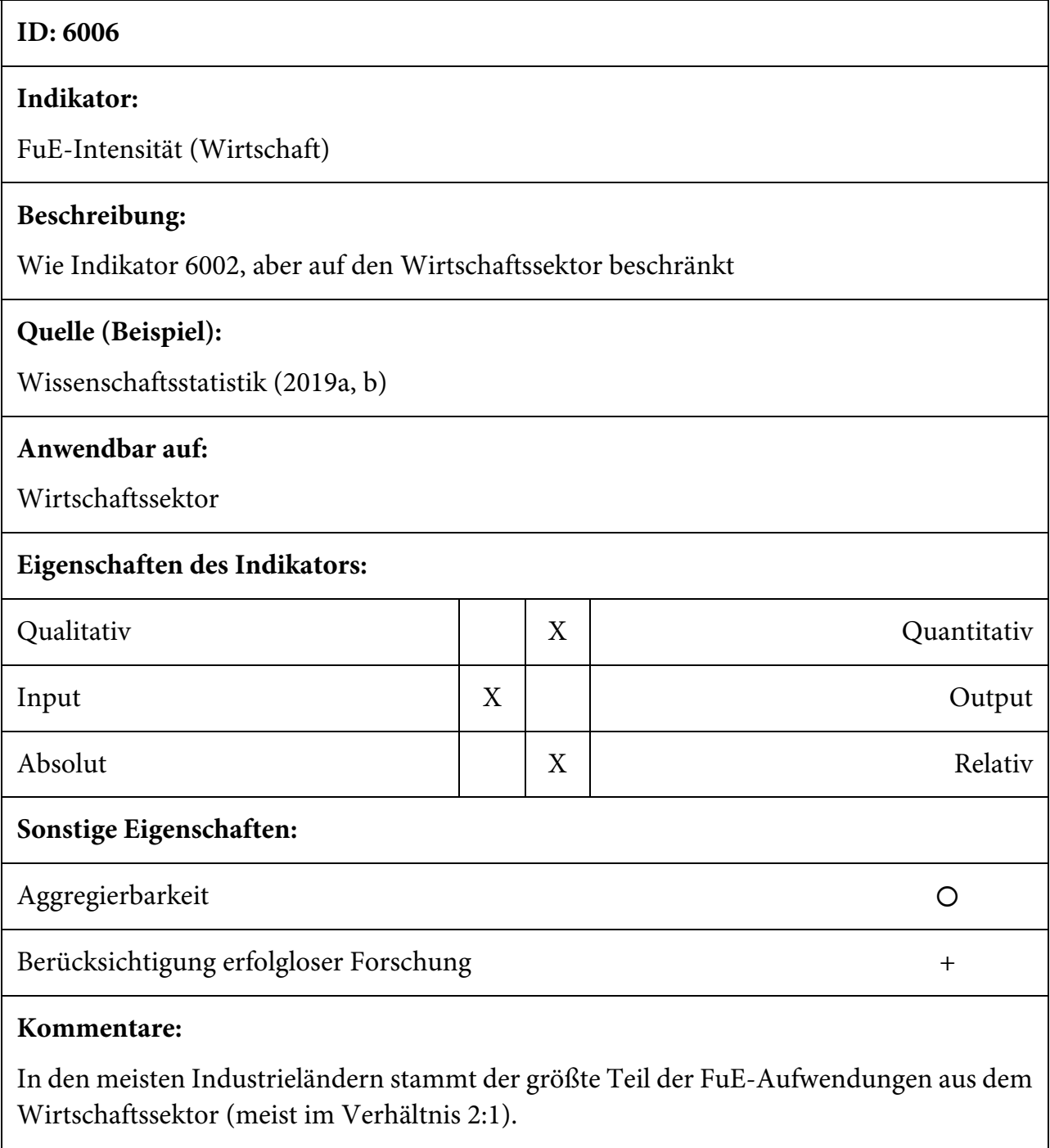


ID: 6007

\section{Indikator:}

Globale FuE-Aufwendungen multinationaler Unternehmen

\section{Beschreibung:}

FuE-Aufwendungen der Unternehmen weltweit

Quelle (Beispiel):

OECD (2015), Kap. 11; Wissenschaftsstatistik (2019a), Tab. 4.3; European Commission (2019)

Anwendbar auf:

Wirtschaftssektor

Eigenschaften des Indikators:

\begin{tabular}{|l|l|l|r|}
\hline Qualitativ & & $\mathrm{X}$ & Quantitativ \\
\hline Input & $\mathrm{X}$ & & Output \\
\hline Absolut & $\mathrm{X}$ & & Relativ \\
\hline
\end{tabular}

Sonstige Eigenschaften:

Aggregierbarkeit

$+$

Berücksichtigung erfolgloser Forschung

$+$

\section{Kommentare:}

Die Differenz zwischen den weltweiten FuE-Aufwendungen und den inländischen FuEAufwendungen der multinationalen Konzerne bezeichnet man als „Outward“-FuE (Indikator 6008). 


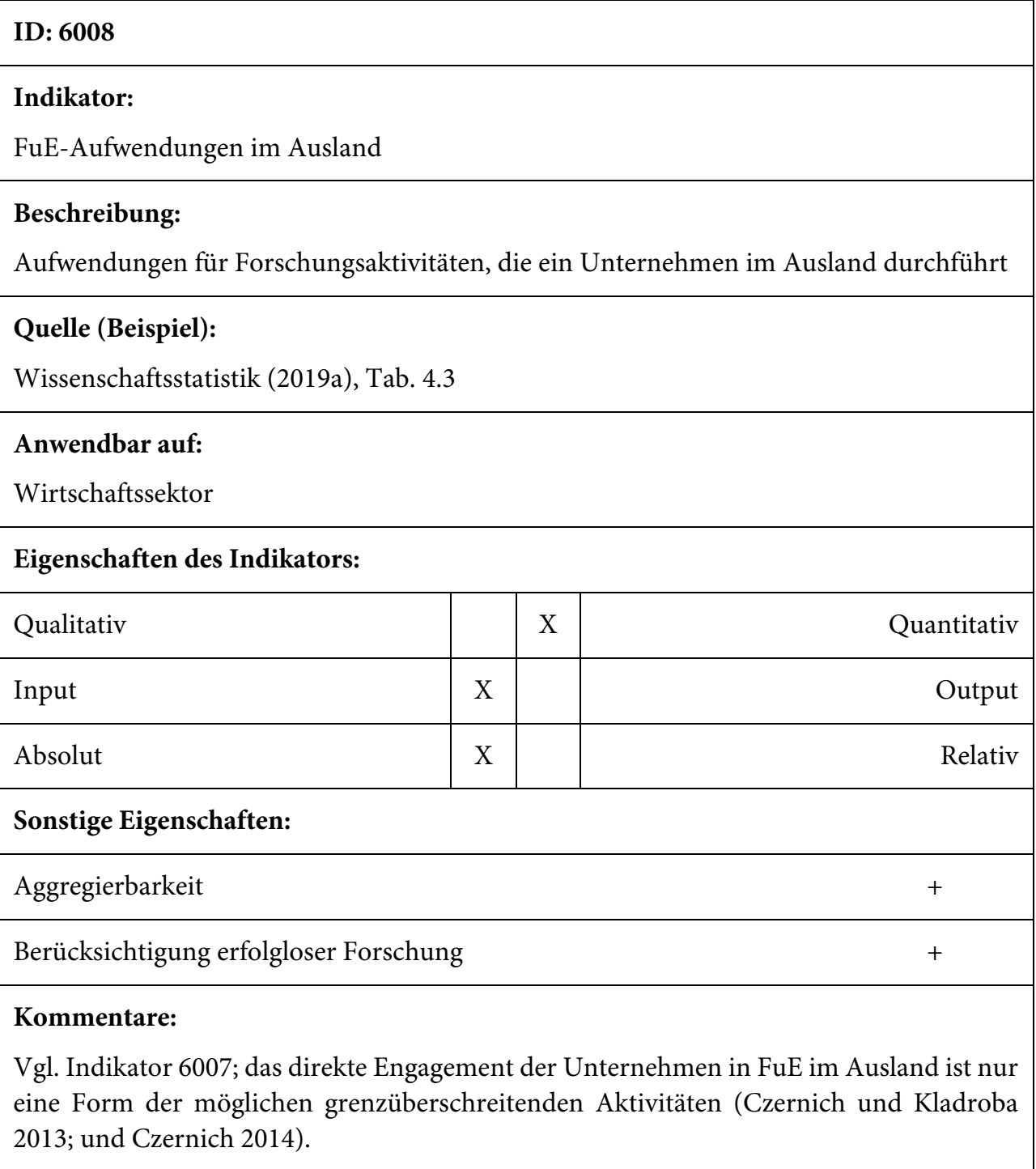




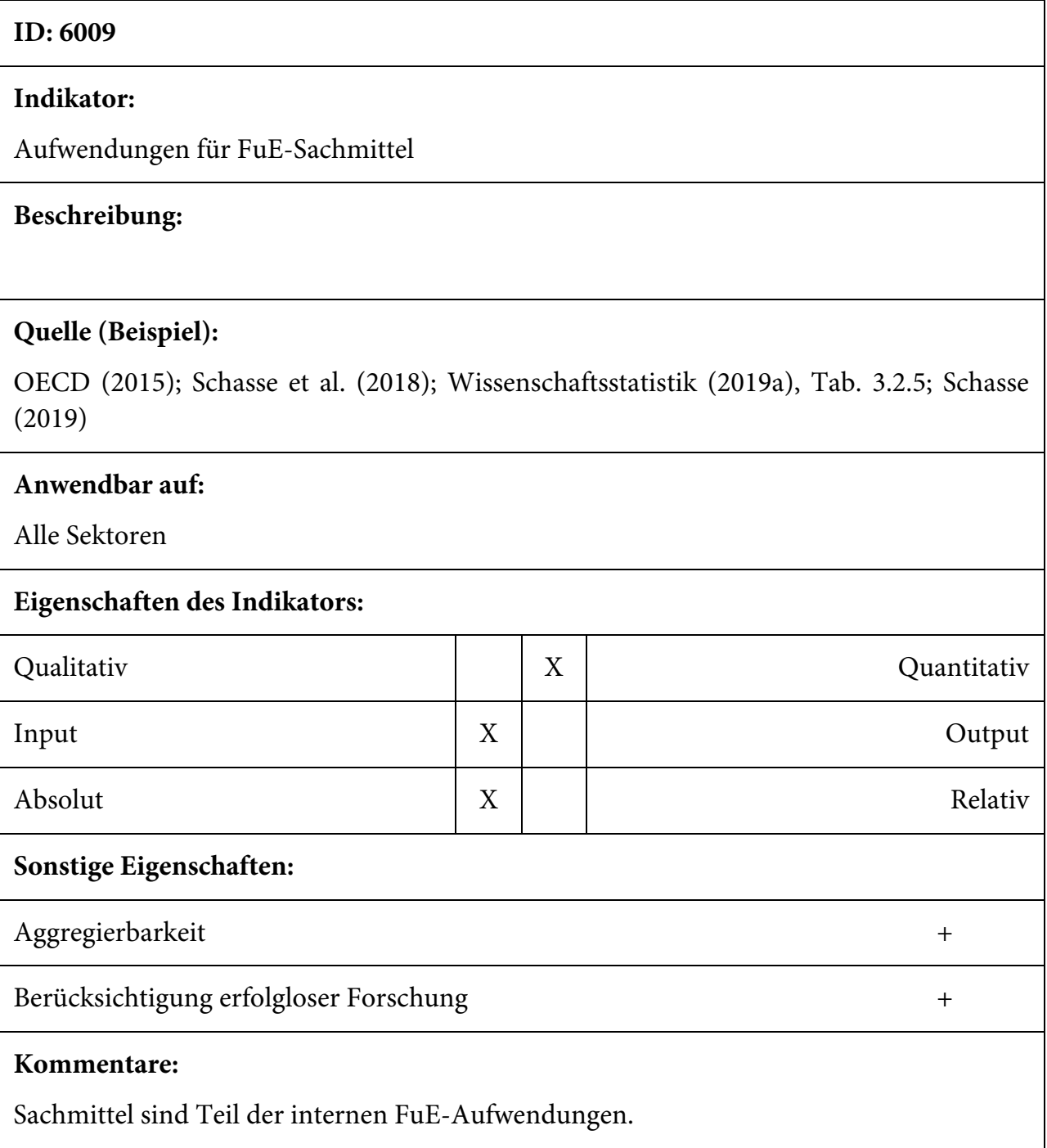




\begin{tabular}{l} 
ID: 6010 \\
\hline $\begin{array}{l}\text { Indikator: } \\
\text { Aufwendungen für FuE-Anlagen }\end{array}$ \\
\hline $\begin{array}{l}\text { Beschreibung: } \\
\text { Einmalige Investitionen in Anlagen, die besonders für FuE genutzt werden (FuE-Investi- } \\
\text { tionen). }\end{array}$ \\
\hline $\begin{array}{l}\text { Quelle (Beispiel): } \\
\text { OECD (2015); Schasse et al. (2018); Wissenschaftsstatistik (2019a), Tab. 3.2.5; Schasse } \\
\text { (2019) }\end{array}$ \\
\hline
\end{tabular}

Anwendbar auf:

Alle Sektoren

Eigenschaften des Indikators:

\begin{tabular}{|l|l|l|r|}
\hline Qualitativ & $\mathrm{X}$ & $\mathrm{X}$ & Quantitativ \\
\hline Input & $\mathrm{X}$ & & Output \\
\hline Absolut & $\mathrm{X}$ & & Relativ \\
\hline
\end{tabular}

Sonstige Eigenschaften:

Aggregierbarkeit + +

Berücksichtigung erfolgloser Forschung +

\section{Kommentare:}

Die FuE-Investitionen sind Teil der internen FuE-Aufwendungen. Die Investitionen werden in der FuE-Statistik im Jahr ihres Anfallens geführt. Im Gegensatz zur VGR gibt es in der FuE-Statistik keine Abschreibungen. 


\section{ID: 6011}

\section{Indikator:}

Anteil der FuE-Aufwendungen an den Innovationsaufwendungen

\section{Beschreibung:}

\section{Quelle (Beispiel):}

OECD (2018); Rammer (2019)

\section{Anwendbar auf:}

Wirtschaftssektor

\section{Eigenschaften des Indikators:}

\begin{tabular}{|l|l|l|l|}
\hline Qualitativ & & $\mathrm{X}$ & Quantitativ \\
\hline Input & $\mathrm{X}$ & & Output \\
\hline Absolut & & $\mathrm{X}$ & Relativ \\
\hline
\end{tabular}

\section{Sonstige Eigenschaften:}

Aggregierbarkeit

Berücksichtigung erfolgloser Forschung

\section{Kommentare:}

Gemäß dem Oslo-Handbuch sind die FuE-Aufwendungen ein Teil der Innovationsaufwendungen eines Unternehmens. Darüber hinaus unterscheidet das Oslo-Handbuch zwischen Innovationen mit und Innovationen ohne FuE. 
ID: 6012

\section{Indikator:}

Staatsausgaben für Bildung, Forschung und Technologie

\section{Beschreibung:}

Umfasst alle Formen von staatlichen Ausgaben (Bund, Länder, Gemeinden) für die drei genannten Bereiche.

\section{Quelle (Beispiel):}

Hotz-Hart und Rohner (2014), S. 17

\section{Anwendbar auf:}

Alle Sektoren, beschreibt aber die Gebersicht (Staat)

Eigenschaften des Indikators:

\begin{tabular}{|l|l|l|r|}
\hline Qualitativ & & $\mathrm{X}$ & Quantitativ \\
\hline Input & $\mathrm{X}$ & & Output \\
\hline Absolut & $\mathrm{X}$ & & Relativ \\
\hline
\end{tabular}

Sonstige Eigenschaften:

Aggregierbarkeit

$+$

Berücksichtigung erfolgloser Forschung

$+$

\section{Kommentare:}

Umfasst auch die internen FuE-Aufwendungen des Staates, geht aber weit darüber hinaus. 
ID: 6013

\section{Indikator:}

FuE-Aufwendungen der Länder

\section{Beschreibung:}

FuE-Aufwendungen nach Bundesländern (NUTS 1); tiefere Gliederungen (NUTS 2) liegen ebenfalls vor.

\section{Quelle (Beispiel):}

BMBF (2020b), S. 16 ff.

\section{Anwendbar auf:}

Alle Sektoren

Eigenschaften des Indikators:

\begin{tabular}{|l|l|l|r|}
\hline Qualitativ & & $\mathrm{X}$ & Quantitativ \\
\hline Input & $\mathrm{X}$ & & Output \\
\hline Absolut & $\mathrm{X}$ & & Relativ \\
\hline
\end{tabular}

Sonstige Eigenschaften:

Aggregierbarkeit

Berücksichtigung erfolgloser Forschung

\section{Kommentare:}

Zur besseren Vergleichbarkeit wird oftmals der Bezug zum regionalen Bruttoinlandsprodukt hergestellt. 


\section{ID: 6014}

\section{Indikator:}

Haushaltsansätze des Staates für FuE

\section{Beschreibung:}

Umfasst die Grundfinanzierung sowie Drittmittel von Hochschulen und Forschungseinrichtungen, aber auch die FuE-Förderung von Unternehmen

\section{Quelle (Beispiel):}

Schasse (2019), Kap. 4

\section{Anwendbar auf:}

Alle Sektoren, die staatliche Zuschüsse für FuE bekommen

\section{Eigenschaften des Indikators:}

\begin{tabular}{|l|l|l|r|}
\hline Qualitativ & & $\mathrm{X}$ & Quantitativ \\
\hline Input & $\mathrm{X}$ & & Output \\
\hline Absolut & $\mathrm{X}$ & & Relativ \\
\hline
\end{tabular}

\section{Sonstige Eigenschaften:}

Aggregierbarkeit

Berücksichtigung erfolgloser Forschung

\section{Kommentare:}

Haushaltsansätze können als Frühindikator für die Entwicklung der FuE-Aufwendungen verwendet werden. Sie stehen bereits zu Beginn des Berichtsjahres zur Verfügung. Letztlich kann es aber zu Differenzen zwischen den Haushaltsansätzen und den tatsächlich realisierten FuE-Aufwendungen kommen. 
ID: 6015

\section{Indikator:}

FuE-Aufwendungen nach Art der Forschung

\section{Beschreibung:}

Unterschieden wird in Grundlagenforschung, angewandte Forschung und experimentelle Entwicklung.

\section{Quelle (Beispiel):}

OECD (2015), Ziffer 1.35 und Kap. 2.5; Wissenschaftsstatistik (2019a), Tab. 3.2.6; Destatis (2020), Tab. 3.8

Anwendbar auf:

Alle Sektoren

Eigenschaften des Indikators:

\begin{tabular}{|l|l|l|r|}
\hline Qualitativ & & $\mathrm{X}$ & Quantitativ \\
\hline Input & $\mathrm{X}$ & & Output \\
\hline Absolut & $\mathrm{X}$ & & Relativ \\
\hline
\end{tabular}

Sonstige Eigenschaften:

Aggregierbarkeit

Berücksichtigung erfolgloser Forschung

Kommentare: 


\section{Hochschulen}

\begin{tabular}{|c|c|c|c|}
\hline \multicolumn{4}{|l|}{ ID: 6016} \\
\hline \multicolumn{4}{|l|}{ Indikator: } \\
\hline \multicolumn{4}{|c|}{ Leistungsbezogene Haushaltsmittel } \\
\hline \multicolumn{4}{|c|}{ Beschreibung: } \\
\hline \multicolumn{4}{|c|}{$\begin{array}{l}\text { Neben den Grundmitteln bekommen Hochschulen und Forschungsinstitute oftmals leis- } \\
\text { tungsabhängige Mittel zugewiesen. }\end{array}$} \\
\hline \multicolumn{4}{|c|}{ Quelle (Beispiel): } \\
\hline \multicolumn{4}{|c|}{ Jäger (2008); Kreysing (2008); Kamm und Krempkow (2010) } \\
\hline \multicolumn{4}{|c|}{ Anwendbar auf: } \\
\hline \multicolumn{4}{|c|}{ Hochschulen, Forschungsinstitute } \\
\hline \multicolumn{4}{|c|}{ Eigenschaften des Indikators: } \\
\hline Qualitativ & & $\mathrm{X}$ & Quantitativ \\
\hline Input & $\mathrm{X}$ & & Output \\
\hline Absolut & $\mathrm{X}$ & & Relativ \\
\hline
\end{tabular}

Sonstige Eigenschaften:

Aggregierbarkeit + +

Berücksichtigung erfolgloser Forschung

$(+)$

\section{Kommentare:}

Leistungsbezogene Haushaltsmittel sind auch als Indikator für Aggregate geeignet, wenn nicht nur die Verteilung der Mittel leistungsbezogen ist, sondern auch die Summe. Voraussetzung ist also, dass nicht nur eine vorher festgelegte Summe nach einem definierten Schlüssel verteilt wird, sondern sich die Summe auch durch gute Leistungen aller Beteiligten erhöhen kann. 
ID: 6017

Indikator:

Anteil der durch Unternehmen finanzierten FuE an Hochschulen

Beschreibung:

Entspricht den speziell für Forschung eingeworbenen Drittmitteln (vgl. auch Kapitel 8)

Quelle (Beispiel):

Schubert et al. (2011), S. 24

Anwendbar auf:

Hochschulen

Eigenschaften des Indikators:

\begin{tabular}{|l|l|l|r|}
\hline Qualitativ & & $\mathrm{X}$ & Quantitativ \\
\hline Input & $\mathrm{X}$ & & Output \\
\hline Absolut & & $\mathrm{X}$ & Relativ \\
\hline
\end{tabular}

Sonstige Eigenschaften:

Aggregierbarkeit

Berücksichtigung erfolgloser Forschung

Kommentare: 


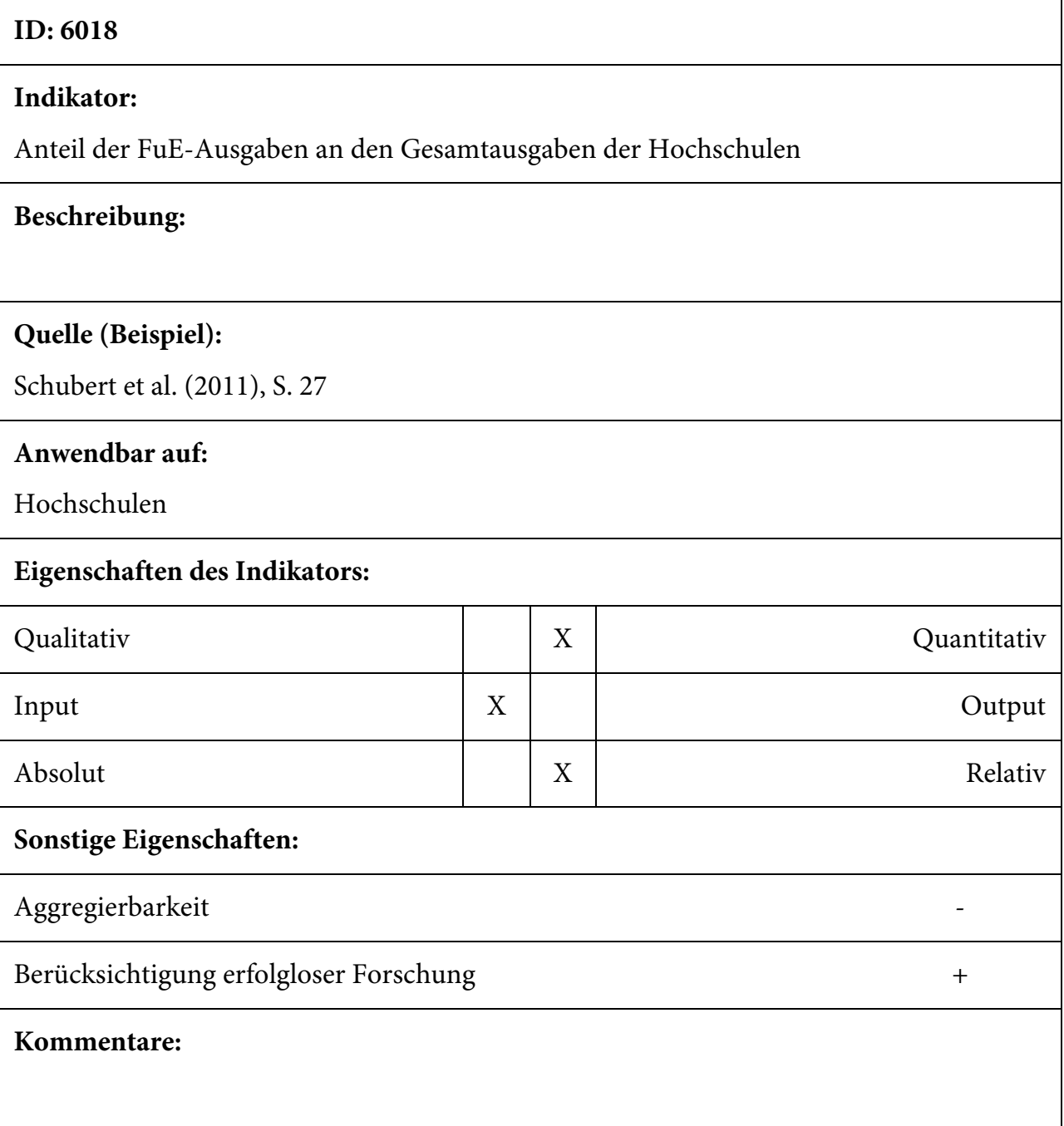




\section{ID: 6019}

\section{Indikator:}

Kosten für Forschungsinfrastruktur

\section{Beschreibung:}

Gemessen wird die übergeordnete Infrastruktur, die allen Forschenden an einer Hochschule gleichermaßen zur Verfügung steht (z. B. Bibliotheken)

\section{Quelle (Beispiel):}

Werner (2002), S. 60

\section{Anwendbar auf:}

Hochschulen

\section{Eigenschaften des Indikators:}

\begin{tabular}{|l|l|l|r|}
\hline Qualitativ & & $\mathrm{X}$ & Quantitativ \\
\hline Input & $\mathrm{X}$ & & Output \\
\hline Absolut & $\mathrm{X}$ & & Relativ \\
\hline
\end{tabular}

\section{Sonstige Eigenschaften:}

Aggregierbarkeit

Berücksichtigung erfolgloser Forschung

\section{Kommentare:}

Vergleichbarkeit wird erreicht, indem die Kosten relativ zur Größe der Hochschule (Anzahl der Studierenden, Anzahl der Professorinnen und Professoren u. Ä.) dargestellt werden. 


\section{FuE-/Innovationsförderung}

ID: 6020

\section{Indikator:}

B-Index für steuerfinanzierte FuE-Subventionen

\section{Beschreibung:}

Barwert der zukünftigen Investitionsrückflüsse, die ein Unternehmen benötigt, um die Kosten für FuE plus Einkommensteuer zu decken.

Quelle (Beispiel):

Schubert et al. (2011), S. 25

Anwendbar auf:

Unternehmen

Eigenschaften des Indikators:

\begin{tabular}{|l|l|l|l|}
\hline Qualitativ & & $\mathrm{X}$ & Quantitativ \\
\hline Input & & $\mathrm{X}$ & Output \\
\hline Absolut & $\mathrm{X}$ & & Relativ \\
\hline
\end{tabular}

Sonstige Eigenschaften:

Aggregierbarkeit

Berücksichtigung erfolgloser Forschung

Kommentare: 
ID: 6021

\section{Indikator:}

Staatlich geförderte FuE im Verhältnis zum nationalen oder regionalen BIP

\section{Beschreibung:}

Gemessen werden die Aufwendungen für die FuE, die in Form von Zuschüssen oder Aufträgen staatlich gefördert wird.

\section{Quelle (Beispiel):}

Schubert et al. (2011), S. 25

\section{Anwendbar auf:}

Alle Sektoren

Eigenschaften des Indikators:

\begin{tabular}{|l|l|l|r|}
\hline Qualitativ & & $\mathrm{X}$ & Quantitativ \\
\hline Input & $\mathrm{X}$ & & Output \\
\hline Absolut & & $\mathrm{X}$ & Relativ \\
\hline
\end{tabular}

Sonstige Eigenschaften:

Aggregierbarkeit

Berücksichtigung erfolgloser Forschung

Kommentare: 
ID: 6022

Indikator:

Abschreibungen auf Forschungsgeräte

\section{Beschreibung:}

FuE-Investitionen werden über ihre Nutzungsdauer abgeschrieben

Quelle (Beispiel):

Werner (2002), S. 60

Anwendbar auf:

Alle Sektoren

Eigenschaften des Indikators:

\begin{tabular}{|l|l|l|l|}
\hline Qualitativ & & $\mathrm{X}$ & Quantitativ \\
\hline Input & $\mathrm{X}$ & & Output \\
\hline Absolut & $\mathrm{X}$ & & Relativ \\
\hline
\end{tabular}

Sonstige Eigenschaften:

Aggregierbarkeit + +

Berücksichtigung erfolgloser Forschung + +

\section{Kommentare:}

Abschreibungen auf FuE-Investitionen sind nicht Teil der FuE-Statistik (vgl. Indikator 6010), können darüber hinaus aber ein ergänzender Indikator für die Entwicklung der FuE-Investitionen sein. 
ID: 6023

\section{Indikator:}

FuE-Aufwendungen nach Herkunft der Mittel

\section{Beschreibung:}

Es wird nach verschiedenen Finanzierungsquellen unterschieden (z. B. selber finanziert, staatlich gefördert, aus dem Ausland finanziert, von einem anderen Sektor finanziert)

\section{Quelle (Beispiel):}

OECD (2015), Kap. 4; Wissenschaftsstatistik (2019a), Abschnitt 3.1

\section{Anwendbar auf:}

Alle Sektoren

\section{Eigenschaften des Indikators:}

\begin{tabular}{|l|l|l|r|}
\hline Qualitativ & & $\mathrm{X}$ & Quantitativ \\
\hline Input & $\mathrm{X}$ & & Output \\
\hline Absolut & $\mathrm{X}$ & & Relativ \\
\hline
\end{tabular}

\section{Sonstige Eigenschaften:}

Aggregierbarkeit

Berücksichtigung erfolgloser Forschung

\section{Kommentare:}

Neben der Durchführung von FuE ist auch die Finanzierung ein wichtiger Indikator. Dabei spielt außer den bekannten Sektoren auch das Ausland (in Anlehnung an die VGR "Rest of the World“) eine wichtige Rolle. 
ID: 6024

\section{Indikator:}

Nutzung der staatlichen Innovationsförderung

\section{Beschreibung:}

In welchem Maße werden staatliche Innovationsfördermaßnahmen durch Unternehmen in Anspruch genommen?

\section{Quelle (Beispiel):}

OECD (2018), S. 210

\section{Anwendbar auf:}

Wirtschaftssektor

Eigenschaften des Indikators:

\begin{tabular}{|l|l|l|r|}
\hline Qualitativ & & $\mathrm{X}$ & Quantitativ \\
\hline Input & $\mathrm{X}$ & & Output \\
\hline Absolut & $\mathrm{X}$ & & Relativ \\
\hline
\end{tabular}

Sonstige Eigenschaften:

Aggregierbarkeit

Berücksichtigung erfolgloser Forschung

$+$

Kommentare: 
ID: 6025

\section{Indikator:}

FuE-Aufwendungen pro Euro Steuergutschrift

\section{Beschreibung:}

Bei Vorliegen einer steuerlichen FuE-Förderung wird der Quotient zwischen FuE-Aufwendungen und aus FuE resultierender Steuergutschrift gebildet.

\section{Quelle (Beispiel):}

Bérubé und Mohnen (2009), S. 207

\section{Anwendbar auf:}

Wirtschaftssektor

\section{Eigenschaften des Indikators:}

\begin{tabular}{|l|l|l|r|}
\hline Qualitativ & & $\mathrm{X}$ & Quantitativ \\
\hline Input & $\mathrm{X}$ & & Output \\
\hline Absolut & & $\mathrm{X}$ & Relativ \\
\hline
\end{tabular}

\section{Sonstige Eigenschaften:}

Aggregierbarkeit

Berücksichtigung erfolgloser Forschung

\section{Kommentare:}

Der Indikator gibt an, in welcher Höhe Steuergutschriften FuE-Aufwendungen generieren. Bérubé und Mohnen (2009) schlagen außerdem die Anzahl der Unternehmen, die Steuergutschriften für FuE nutzen, sowie die Anzahl der geförderten Unternehmen insgesamt als Indikatoren vor (Indikatoren 6026 und 6027). 
ID: 6026

Indikator:

Anzahl der Unternehmen, die Steuergutschriften auf FuE nutzen

Beschreibung:

Quelle (Beispiel):

Bérubé und Mohnen (2009), S. 208

Anwendbar auf:

Wirtschaftssektor

Eigenschaften des Indikators:

\begin{tabular}{|l|l|l|r|}
\hline Qualitativ & & $\mathrm{X}$ & Quantitativ \\
\hline Input & $\mathrm{X}$ & & Output \\
\hline Absolut & $\mathrm{X}$ & & Relativ \\
\hline
\end{tabular}

Sonstige Eigenschaften:

Aggregierbarkeit +

Berücksichtigung erfolgloser Forschung +

Kommentare: 


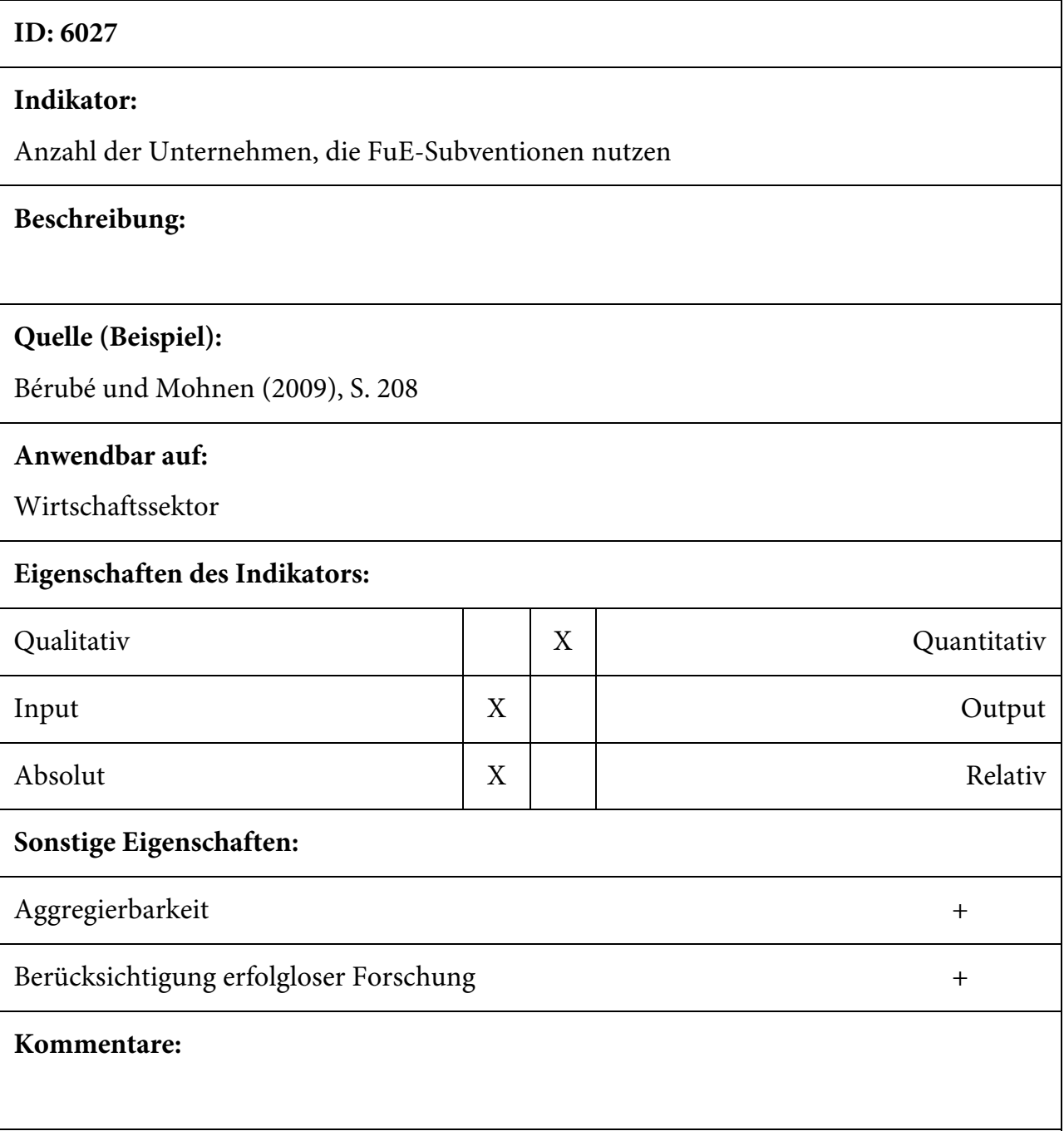




\section{Output-Indikatoren}

ID: 6028

\section{Indikator:}

Umsatzanteil durch neue und verbesserte Produkte

\section{Beschreibung:}

Welcher Umsatz wird durch in den letzten X Jahren neu entwickelte oder verbesserte Produkte im Verhältnis zum Gesamtumsatz generiert?

Quelle (Beispiel):

Wissenschaftsstatistik (2010), S. $45 \mathrm{ff}$.

Anwendbar auf:

Wirtschaftssektor

Eigenschaften des Indikators:

\begin{tabular}{|l|c|c|c|}
\hline Qualitativ & & $\mathrm{X}$ & Quantitativ \\
\hline Input & & $\mathrm{X}$ & Output \\
\hline Absolut & & $\mathrm{X}$ & Relativ \\
\hline
\end{tabular}

Sonstige Eigenschaften:

Aggregierbarkeit

Berücksichtigung erfolgloser Forschung

\section{Kommentare:}

Eine zusätzliche Information besteht darin, ob der Innovation, die zur Umsatzsteigerung geführt hat, FuE zugrunde lag oder nicht. 


\section{Negativindikatoren}

ID: 6029

\section{Indikator:}

Gründe, auf Innovationen zu verzichten

\section{Beschreibung:}

Aufgezählt werden die Gründe, die eine Innovation verhindert haben (z. B. gesetzliche Vorgaben, Fachkräftemangel usw.)

Quelle (Beispiel):

Rammer (2018), S. 68

Anwendbar auf:

Wirtschaftssektor

Eigenschaften des Indikators:

\begin{tabular}{|l|c|c|r|}
\hline Qualitativ & $\mathrm{X}$ & & Quantitativ \\
\hline Input & $\mathrm{X}$ & & Output \\
\hline Absolut & $\mathrm{X}$ & & Relativ \\
\hline
\end{tabular}

Sonstige Eigenschaften:

Aggregierbarkeit +

Berücksichtigung erfolgloser Forschung

Kommentare:

Die Innovationserhebung des ZEW hat für das Jahr 2017 danach gefragt, welche Hemmnisse oder sogar Verzichtsgründe für Innovationsaktivitäten vorlagen (und deren Bedeutung). Darüber hinaus könnte eine interessante Fragestellung sein, wie hoch die Ausgaben für die Innovationen gewesen wären, die dadurch verloren gegangen sind. 


\section{Literatur}

Bérubé, C., \& Mohnen, P. (2009). Are Firms That Receive R\&D Subsidies More Innovative? Canadian Journal of Economics, 42, 206-225.

BMBF (2020a). Bundesbericht Forschung und Innovation 2020 - Forschungs- und innovationspolitische Ziele und Maßnahmen (Hauptband). Berlin: BMBF.

BMBF (2020b). Daten und Fakten zum deutschen Forschungs- und Innovationssystem. Berlin: BMBF.

Czernich, N., \& Kladroba, A. (2013). Forschung und Entwicklung in international agierenden Unternehmen. In Wissenschaftsstatistik (Hrsg.), FuE-Datenreport 2013 (S. 33-38). Essen. http://stifterverband.de/pdf/fue_datenreport_2013_analysen_und_vergleiche.pdf. Zugegriffen: 18. Sep. 2020

Czernich, N. (2014). Forschung und Entwicklung deutscher Unternehmen im Ausland - Zielländer, Motive und Schwierigkeiten. Studien zum deutschen Innovationssystem 14-2014. Berlin. https:// www.e-fi.de/fileadmin/Innovationsstudien_2014/StuDIS_13_2014.pdf. Zugegriffen: 18. Sep. 2020

Destatis (2020). Ausgaben, Einnahmen und Personal der öffentlichen und öffentlich geförderten Einrichtungen für Wissenschaft, Forschung und Entwicklung. Fachserie 14, Reihe 3.6. Wiesbaden: Destatis.

European Commission (2019). The 2019 EU Industrial R\&D Scoreboard. Brüssel. https://op.europa.eu/en/publication-detail/-/publication/bcbeb233-216c-11ea-95ab-01aa75ed71a1/languageen. Zugegriffen: 18. Sep. 2020

Hotz-Hart, B., \& Rohner, A. (2014). Nationen im Innovationswettlauf: Ökonomie und Politik der Innovation. Wiesbaden: Springer Gabler.

Jäger, M. (2008). Wie wirksam sind leistungsorientierte Budgetierungsverfahren an deutschen Hochschulen? Zeitschrift für Hochschulentwicklung, 3, 89-104.

Kramm, R., \& Krempkow, R. (2010). Ist leistungsorientierte Mittelvergabe im Hochschulbereich ,gerecht" gestaltbar? Qualität in der Wissenschaft, 3, 71-78.

Kreysing, M. (2008). Forschungsförderung mittels leistungsorientierter Mittelvergabe. Zeitschrift für Hochschulentwicklung, 3, 19-28.

OECD (2015). Frascati Manual 2015: Guidelines for Collecting and Reporting Data on Research and Experimental Development. Paris: OECD Publishing.

OECD (2018). Oslo Manual 2018: Guidelines for Collecting, Reporting and Using Data on Innovation. Paris: OECD Publishing.

Rammer, C. (2018). Dokumentation zur Innovationserhebung 2017. Dokumentation Nr. 18-01. Mannheim: ZEW. http://ftp.zew.de/pub/zew-docs/docus/dokumentation1801.pdf. Zugegriffen: 18. Sep. 2020

Rammer, C. (2019). Dokumentation zur Innovationserhebung 2018, Dokumentation Nr. 19-01. Mannheim: ZEW. http://ftp.zew.de/pub/zew-docs/docus/dokumentation1901.pdf. Zugegriffen: 18. Sep. 2020

Schasse, U. (2019). Forschung und Entwicklung in Staat und Wirtschaft: Kurzstudie 2019. Studien zum deutschen Innovationssystem Nr. 2-2019. https://www.e-fi.de/fileadmin/Innovationsstudien 2019/StuDIS_02_2019.pdf. Zugegriffen: 18. Sep. 2020

Schasse, U., Gehrke, B., \& Stenke, G. (2018). Forschung und Entwicklung in Staat und Wirtschaft: Deutschland im internationalen Vergleich. Studien zum deutschen Innovationssystem 2-2018. https://www.e-fi.de/fileadmin/Innovationsstudien_2018/StuDIS_02_2018.pdf. Zugegriffen: 18. Sep. 2020

Schubert, T., Neuhäusler, P., Frietsch, R., Rammer, C., \& Hollanders, H. (2011). Innovation Indicator Methodology Report. http://www.innovationsindikator.de/fileadmin/content/2018/pdf/methodology_report.pdf. Zugegriffen: 18. Sep. 2020 
Werner, B. M. (2002). Messung und Bewertung der Leistung von Forschung und Entwicklung im Innovationsprozess. Dissertation. TU Darmstadt. http://tuprints.ulb.tu-darmstadt.de/200/. Zugegriffen: 18. Sep. 2020

Wissenschaftsstatistik (2010). Datenreport 2010. Essen. http://www.stifterverband.de/pdf/fue_datenreport_2010.pdf. Zugegriffen: 18. Sep. 2020

Wissenschaftsstatistik (2019a). ARENDI Zahlenwerk. Essen. https://stifterverband.org/download/file/ fid/7762. Zugegriffen: 18. Sep. 2020

Wissenschaftsstatistik (2019b). ARENDI Analysen. Essen. https://www.stifterverband.org/download/ file/fid/8062. Zugegriffen: 18. Sep. 2020

Open Access Dieses Kapitel wird unter der Creative Commons Namensnennung 4.0 International Lizenz (http://creativecommons.org/licenses/by/4.0/deed.de) veröffentlicht, welche die Nutzung, Vervielfältigung, Bearbeitung, Verbreitung und Wiedergabe in jeglichem Medium und Format erlaubt, sofern Sie den/die ursprünglichen Autor(en) und die Quelle ordnungsgemäß nennen, einen Link zur Creative Commons Lizenz beifügen und angeben, $\mathrm{ob}^{-}$ Änderungen vorgenommen wurden.

Die in diesem Kapitel enthaltenen Bilder und sonstiges Drittmaterial unterliegen ebenfalls der genannten Creative Commons Lizenz, sofern sich aus der Abbildungslegende nichts anderes ergibt. Sofern das betreffende Material nicht unter der genannten Creative Commons Lizenz steht und die betreffende Handlung nicht nach gesetzlichen Vorschriften erlaubt ist, ist für die oben aufgeführten Weiterverwendungen des Materials die Einwilligung des jeweiligen Rechteinhabers einzuholen. 